\title{
Baicalein Preconditioning Modulates Hepatocellular Injury following Liver Ischemia and Reperfusion in Rats via Anti-Inflammatory and Antioxidant Signaling
}

\author{
Mona Fouad Mahmoud Samar Gamal Hassan M. El-Fayoumi \\ Department of Pharmacology and Toxicology, Faculty of Pharmacy, Zagazig University, Zagazig, Egypt
}

\begin{abstract}
Keywords
Hepatic ischemia/reperfusion - Baicalein - Antioxidants .

Anti-inflammatory agents - Ischemia/reperfusion injury .

Liver - Caspase
\end{abstract}

\begin{abstract}
Background: Hepatic ischemia/reperfusion (I/R) is a frequent and major complication during liver transplantation. Baicalein, a plant flavonoid, has anti-inflammatory and antioxidant effects. However, whether these effects mediate its protective effects in liver I/R injury remains poorly understood. Objective: This study was designed to investigate the effects of baicalein preconditioning on liver $\mathrm{I} / \mathrm{R}$ in rats and the underlying mechanisms. Methods: Baicalein $(300 \mathrm{mg} /$ $\mathrm{kg}$ ) was intraperitoneally injected $30 \mathrm{~min}$ before $45 \mathrm{~min}$ of ischemia followed by $1 \mathrm{~h}$ of reperfusion. Results: Baicalein preconditioning attenuated liver I/R injury, as indicated by a reduction in serum aminotransferase activities and an improvement of histopathological abnormalities. Baicalein also significantly reduced the activity of the cellular hepatic proapoptotic enzyme caspase-3 in response to I/R injury. Moreover, baicalein significantly reduced nuclear factor kappa-B expression and the subsequent proinflammatory cyto-
\end{abstract}

\begin{tabular}{ll}
\hline KARGER & ( 2019 The Author(s) \\
& Published by S. Karger AG, Basel \\
E-Mail karger@karger.com & This article is licensed under the Creative Commons Attribution- \\
www.karger.com/dmj & NonCommercial-NoDerivatives 4.0 International License (CC BY- \\
NC-ND) (http://www.karger.com/Services/OpenAccessLicense). \\
Usage and distribution for commercial purposes as well as any dis- \\
tribution of modified material requires written permission.
\end{tabular}

kine production, tumor necrosis factor alpha (TNF-a) level, increased interleukin-10 (IL-10), an anti-inflammatory cytokine, reduced the TNF-a/IL-10 ratio, and suppressed leukocyte infiltration. It increased hepatic antioxidants and reduced lipid peroxidation. Conclusion: Taken together, baicalein preconditioning would seem to protect against liver I/R injury via antioxidant, antiapoptotic, and anti-inflammatory effects.

(C) 2019 The Author(s)

Published by S. Karger AG, Basel

\section{Introduction}

Liver transplantation is the only established treatment for acute liver failure caused by acute drug toxicity, viral hepatitis, or end-stage liver disease. Hepatic ischemia/reperfusion (I/R) is a frequent and major complication during liver transplantation. It is a series of complicated cellular events that occur upon restoration of hepatic blood flow (reperfusion) after a period of ischemia [1]. There are multiple mechanisms that are involved in the pathophysiology of I/R injury, one of them involving the deleterious effect of reactive oxygen species (ROS) and other free radicals. In addition, inflammatory reactions are mediated 
through transcription of proinflammatory cytokines such as tumor necrosis factor alpha (TNF- $\alpha$ ) and interleukin (IL-1, IL-6) [2-4]. Previous studies have demonstrated that oxidative stress and inflammation play an important role in the pathogenesis of $I / R$ [2]. Therefore, the use of antioxidants or anti-inflammatory agents may hinder or even prevent the deleterious effects of I/R injury.

Baicalein (5,6,7-trihydroxyflavone) is one of the major flavonoids originally extracted from the roots of the traditional Chinese herbal medicine Huangqin (Scutellaria baicalensis Georgi). Baicalein is a potent antioxidant and free radical scavenger (hydroxyl radicals) and has been regarded as a specific 12/15-lipoxygenase inhibitor and a good xanthine oxidase inhibitor [5-9]. Baicalein also has anti-inflammatory properties since it has been shown to antagonize the expression of adhesion molecules induced by IL- $1 \beta$ and TNF- $\alpha$ [10].

Previous studies showed baicalein to exert a protective effect on neurons against neuronal injury secondary to ischemia insult $[11,12]$ through the inhibition of inflammatory mediators and ROS [13]. Furthermore, a recent study showed that baicalein injection $30 \mathrm{~min}$ before reperfusion promoted recovery from renal injury and alleviated kidney injury in a rat model of renal I/R via antioxidant and anti-inflammatory effects [14]. Previous studies also demonstrated that baicalein has hepatoprotective effects on acute liver failure induced by D-galactosamine/lipopolysaccharide [15]. Moreover, a recent study by Liu et al. [16] showed that baicalein pretreatment protects against hepatic I/R injury in mice via an antiinflammatory effect. Thus, the present study aimed to investigate the effects of baicalein preconditioning on liver $\mathrm{I} / \mathrm{R}$ in rats and the underlying mechanisms.

\section{Materials and Methods}

\section{Drugs and Reagents}

Baicalein was purchased from Shannxi, China. Its purity was 98\% tested by high-performance liquid chromatography. Dimethyl sulfoxide (DMSO) and other chemicals were purchased from Sigma-Aldrich.

\section{Animals}

Twenty-four adult male Wistar rats (200-220 g; Zagazig University, Zagazig, Egypt) were used in the current study. All rats were kept on a 12-h light/dark regime, with free access to food and water.

\section{Study Design}

Rats were randomly assigned to three groups (8 rats in each group), and drug or solvent was administrated intraperitoneally $30 \mathrm{~min}$ before ischemia. All rats were anesthetized by intraperitoneal injection of ketamine ( $75 \mathrm{mg} / \mathrm{kg}$ ). Group 1 rats (sham group) received an equal volume of DMSO vehicle and were then anesthetized; the portal vein and bile duct were exposed but not occluded. Group 2 rats (I/R group) received an equal volume of DMSO and were then subjected to partial liver ischemia (70\%) followed by reperfusion. Ischemia was induced by occluding the hepatic portal vein and the bile duct with a traumatic vascular clamp for $45 \mathrm{~min}$, then the clamp was removed to start reperfusion for $1 \mathrm{~h}$. Group 3 rats (baicalein group) received $300 \mathrm{mg} / \mathrm{kg}$ baicalein dissolved in DMSO and were then intraperitoneally injected a single dose $30 \mathrm{~min}$ before ischemia. Although each experimental group consisted of 8 rats, statistical analysis was performed on 6 rats as 2 rats died during the surgical procedure.

\section{Sample Collection}

Blood was collected from the retro-orbital plexus and centrifuged $\left(3,000 \mathrm{~g}, 4^{\circ} \mathrm{C}, 20 \mathrm{~min}\right)$ for separation of serum. The serum was used to analyze liver enzymes (alanine aminotransferase [ALT], aspartate aminotransferase [AST], and gamma-glutamyl transferase [GGT]) and lactate dehydrogenase (LDH) enzyme. Then animals were sacrificed, and the liver was separated and washed off with cold saline. Livers were divided into two parts: one part was immediately flash-frozen in liquid nitrogen and kept at $-80^{\circ} \mathrm{C}$ for measurement of tissue parameters, and the other part was kept in $10 \%$ formalin for histopathological examination.

\section{Biochemical Analysis}

The serum ALT, AST, and GGT enzyme activities as well as LDH level were measured using commercially available analytical kits (Biodiagnostic Co., Egypt).

\section{Determination of Inflammatory and Anti-Inflammatory}

Markers

The TNF- $\alpha$ level in liver homogenate was detected by ELISA according to the method of Aggarwal and Vilcek [17] using an ELISA kit (Quantikine, USA). Briefly, $50 \mu \mathrm{L}$ of assay diluent was added to each well. Then, $50 \mu \mathrm{L}$ of standard, control, or sample was added. The plate was covered with a plate sealer and incubated at room temperature for $2 \mathrm{~h}$. Then we aspirated each well and washed it, repeating the process four times for a total of five washes. We added $100 \mu \mathrm{L}$ of conjugate to each well. Then we covered it with a new plate sealer and incubated it at room temperature for $2 \mathrm{~h}$. After that it was aspirated and washed five times. We added $100 \mu \mathrm{L}$ of substrate solution to each well and incubated it at room temperature for $30 \mathrm{~min}$. Finally, $100 \mu \mathrm{L}$ of stop solution was added to each well. The absorbance was read at $450 \mathrm{~nm}$ within $30 \mathrm{~min}$. The IL-10 level in liver homogenate was detected similarly by ELISA according to the method of Moore et al. [18] using an ELISA kit (Bio Vendor, Germany).

Determination of Nuclear Factor Kappa-B (NF- $\kappa B)$ by qRT-PCR

Total RNA was extracted from liver tissue homogenate using the SV Total RNA Isolation system (Promega, Madison, WI, USA) according to the manufacturer's protocol. The extracted RNA was reverse transcribed into cDNA using an RT-PCR kit (Stratagene, USA) according to the manufacturer's instruction. The real-time PCR reaction mixture was composed of $25 \mathrm{~mL}$ SYBR Green Mix $(2 \times), 0.5 \mathrm{~mL}$ cDNA, $2 \mathrm{~mL}$ of each primer pair mix $(5 \mathrm{pmol} / \mathrm{mL}$ each primer), and $\mathrm{H}_{2} \mathrm{O}$ to $50 \mathrm{~mL}$. The PCR program used to amplify cDNA consisted of $120 \mathrm{~s}$ at $50^{\circ} \mathrm{C}, 10 \mathrm{~min}$ at $95^{\circ} \mathrm{C}$, and 40 cycles of $15 \mathrm{~s}$ at $94^{\circ} \mathrm{C}, 30 \mathrm{~s}$ at $60^{\circ} \mathrm{C}$ and $30 \mathrm{~s}$ at $72^{\circ} \mathrm{C}$, followed by $10 \mathrm{~min}$ at 

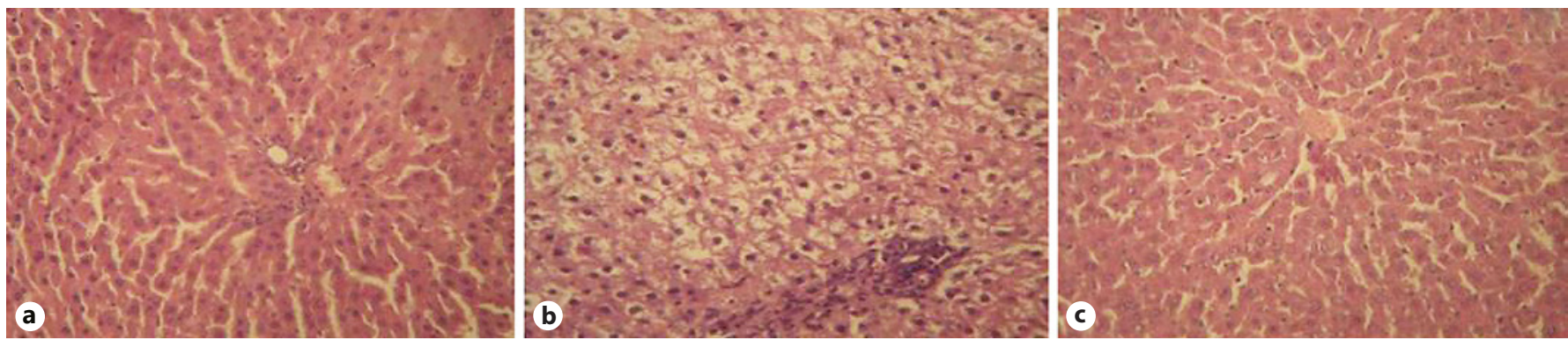

Fig. 1. Liver histology (hematoxylin and eosin staining, original magnification $\times 200$ for all groups) showing representative images of cross sections from liver tissue of 6 rats from different groups. a The sham group showed normal hepatocytes arranged in cords radiating from the central vein. No inflammatory activ- ity could be seen. $\mathbf{b}$ I/R operated rats presented marked hepatocyte degeneration, pyknosis, sinusoidal congestion, and neutrophil infiltration. c The baicalein group showed less pyknosis, reduced hepatocyte degeneration, and cellular infiltrates. I/R, ischemia/reperfusion.

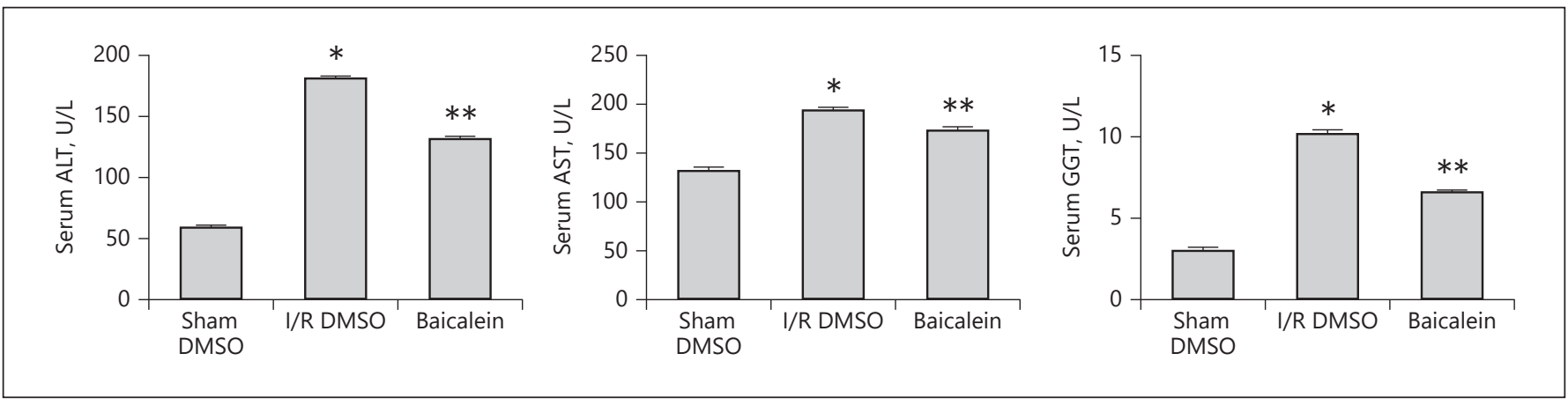

Fig. 2. Effect of I/R-induced liver injury and intraperitoneal administration of baicalein $(300 \mathrm{mg} / \mathrm{kg}$, single dose) on liver enzymes (serum ALT, AST, and GGT activities) $(n=6) .{ }^{*} p<0.05$ (significantly different from the sham group by one-way ANOVA and Tukey's post hoc test); ${ }^{* *} p<0.05$ (significantly different from

$72^{\circ} \mathrm{C}$. The real-time PCR result was analyzed with the step PEApplied Biosystems (Perkin Elmer) software and the data were expressed in cycle threshold. Target gene expression was assessed and related to reference gene ( $\beta$-actin) [19].

\section{Determination of Oxidative Stress Markers}

The generation of ROS in response to hepatic I/R injury was evaluated in liver tissues by the measurement of manganese superoxide dismutase (MnSOD) content, glutathione peroxidase (GPx), and the lipid peroxidation product malondialdehyde (MDA). MDA, MnSOD, and GPx were measured in tissue homogenate photometrically (spectrophotometer, Jenway ${ }^{\circledR}, \mathrm{UK}$ ) according to Nishikimi et al. [20] and Ohkawa et al. [21], respectively.

\section{Determination of the Apoptotic Marker Caspase-3}

Caspase- 3 activity was quantified by proteolytic cleavage of the fluorogenic substrate 7-amino-4-trifluro-methylcoumarin-conjugated Asp-Glu-Val-Asp tetrapeptide (AMC-DEVD) [22]. Liver tissue $(20 \mathrm{mg})$ was homogenized in lysis buffer at $4{ }^{\circ} \mathrm{C}$. After freezing and thawing four times, the lysates were centrifuged at the I/R group by one-way ANOVA and Tukey's post hoc test). ALT, alanine aminotransferase; AST, aspartate aminotransferase; DMSO, dimethyl sulfoxide; GGT, gamma-glutamyl transferase; $\mathrm{I} / \mathrm{R}$, ischemia/reperfusion.

15,000 rpm for $10 \mathrm{~min}$ and the supernatant was collected. Then caspase-3 activity was measured with a fluorescence spectrophotometer (Hitachi F-3010, Japan) using a caspase-3 fluorescence kit (Sigma Co., USA) according to the manufacturer's instructions. The caspase- 3 activity unit was nmol AMC release/h/mg liver tissue [23].

\section{Histopathological Examination}

Liver tissues were fixed in $10 \%$ formalin and embedded in paraffin. Sections were stained with hematoxylin and eosin and then examined under a light microscope for determination of histopathological changes. The histological analysis was performed by a person blinded to treatment (Fig. 1).

\section{Statistical Analysis}

Values were expressed as mean \pm standard deviation. To analyze the differences between groups, statistical analysis was performed by one-way ANOVA followed by Tukey's post hoc test using a computer software (GraphPad, Prism 5, USA). A $p$ value $<0.05$ was considered significant. 
Table 1. Effect of I/R-induced liver injury and intraperitoneal administration of baicalein (300 mg/kg, single dose) on histopathological changes in rats

\begin{tabular}{llll}
\hline Histopathological appearance & Sham & I/R & Baicalein \\
& DMSO & DMSO & \\
\hline Hepatocyte degeneration & - & +++ & + \\
Pyknosis & - & ++++ & + \\
Sinusoidal congestion & - & +++ & + \\
Neutrophil infiltration & - & +++ & +
\end{tabular}

Scoring was done as follows: - , absent; + , mild; ++, moderate; +++ , severe; ++++ , extremely severe. DMSO, dimethyl sulfoxide; $\mathrm{I} / \mathrm{R}$, ischemia/reperfusion.

\section{Results}

\section{Effect of Baicalein on Liver Enzymes}

The serum ALT, AST, and GGT enzyme activities in the different groups are shown in Figure 2. At $60 \mathrm{~min}$ after reperfusion, serum ALT, AST, and GGT enzyme activities were significantly higher in the I/R group than in the sham group. Pretreatment with baicalein showed a significant decrease in the activities of serum ALT, AST, and GGT compared to the I/R group $(p<0.05)$.

\section{Effect of Baicalein on Histopathological Changes}

Histopathological examination of hepatic tissues confirmed the serum transaminases estimation of liver damage. Severe liver injury indicated by hepatocellular degeneration, pyknosis, sinusoidal congestion, and neutrophil infiltration was present in the I/R group compared to the sham group, which was normal. Baicalein preconditioning showed less damage and more recovery from I/R injury (Table 1).

\section{Effect of Baicalein on Inflammatory Response}

Effect of Baicalein on NF- $\kappa B$ Expression. The expression of NF- $\kappa \mathrm{B}$ in the livers of experimental rats was examined by real-time RT-PCR. The mRNA expression of $\mathrm{NF}-\kappa \mathrm{B}$ was significantly increased in the livers of rats in the $\mathrm{I} / \mathrm{R}$ group when compared to the sham group. A significant reduction in NF- $\kappa \mathrm{B}$ mRNA expression level in the baicalein preconditioning group was observed when compared to the I/R group ( $p<0.05$; Fig. 3 ).

Effect of Baicalein on TNF- $\alpha$. The level of the inflammatory marker TNF- $\alpha$ in the livers of experimental rats was examined by ELISA. Compared to the sham group,
Table 2. Effect of I/R-induced liver injury and intraperitoneal administration of baicalein (300 mg/kg, single dose) on liver inflammatory markers (TNF- $\alpha$, TNF- $\alpha /$ IL-10 ratio) and anti-inflammatory mediator (IL-10) in liver tissue $(n=6)$

\begin{tabular}{lccr}
\hline Parameter & Sham & \multicolumn{1}{c}{ I/R } & \multicolumn{1}{c}{ Baicalein } \\
\hline TNF- $\alpha, \mathrm{pg} / \mathrm{mg}$ & $29 \pm 4.2$ & $101 \pm 4.7^{*}$ & $44 \pm 5.9^{* *}$ \\
IL-10, pg/mg & $123 \pm 8.6$ & $67 \pm 4.4^{*}$ & $95 \pm 9.1^{* *}$ \\
TNF- $\alpha / \mathrm{IL}-10$ & $0.24 \pm 0.02$ & $1.51 \pm 0.15^{*}$ & $0.47 \pm 0.1^{* *}$ \\
\hline
\end{tabular}

Results are presented as mean \pm standard deviation. $\mathrm{I} / \mathrm{R}$, ischemia/reperfusion; IL-10, interleukin-10; TNF- $\alpha$, tumor necrosis factor alpha. ${ }^{*} p<0.05$ (significantly different from the sham group by one-way ANOVA and Tukey's post hoc test); ${ }^{* *} p<0.05$ (significantly different from the I/R group by one-way ANOVA and Tukey's post hoc test).

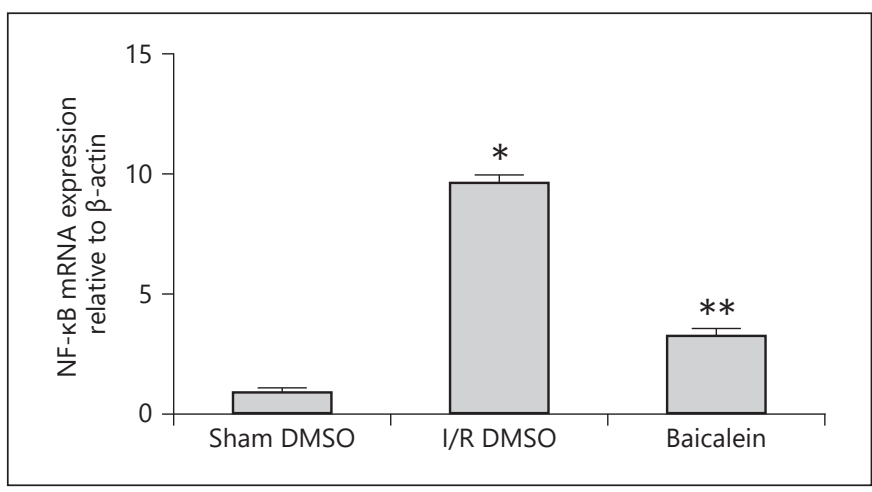

Fig. 3. Effect of I/R-induced liver injury and intraperitoneal administration of baicalein $(300 \mathrm{mg} / \mathrm{kg}$, single dose) on liver NF- $\mathrm{kB}$ gene expression in rats subjected to I/R $(n=6) .{ }^{*} p<0.05$ (significantly different from the sham group by one-way ANOVA and Tukey's post hoc test); ${ }^{* *} p<0.05$ (significantly different from the I/R group by one-way ANOVA and Tukey's post hoc test). DMSO, dimethyl sulfoxide; I/R, ischemia/reperfusion; NF- $\mathrm{B}$, nuclear factor kappa-B.

the TNF- $\alpha$ level was significantly increased in the livers of rats in the I/R group. Compared to the I/R group, a significant reduction in TNF- $\alpha$ level was observed in the baicalein preconditioning group ( $p<0.05$; Table 2$)$.

Effect of Baicalein on IL-10. The level of the anti-inflammatory marker IL-10 in the livers of experimental rats was examined by ELISA. Compared to the sham group, the IL-10 level was significantly decreased in the livers of rats in the I/R group. Compared to the I/R group, a significant elevation of IL-10 level was observed in the baicalein preconditioning group $(p<0.05$; Table 2). 

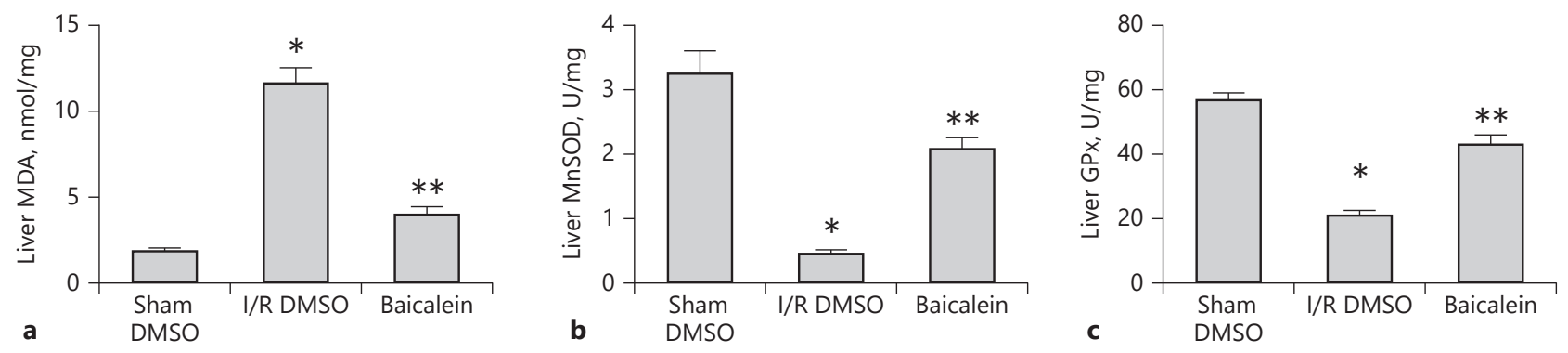

Fig. 4. Effect of I/R-induced liver injury and intraperitoneal administration of baicalein (300 mg/kg, single dose) on liver MDA content (a), MnSOD activity (b), and GPx (c) activity in rats subjected to I/R $(n=6) .{ }^{*} p<0.05$ (significantly different from the sham group by one-way ANOVA and Tukey's post hoc test);
${ }^{* *} p<0.05$ (significantly different from the I/R group by one-way ANOVA and Tukey's post hoc test). DMSO, dimethyl sulfoxide; $\mathrm{GPx}$, glutathione peroxidase; I/R, ischemia/reperfusion; MDA, malondialdehyde; MnSOD, manganese superoxide dismutase.
Fig. 5. Effect of I/R-induced liver injury and intraperitoneal administration of baicalein (300 mg/kg, single dose) on liver caspase-3 (a) and serum LDH activities (b) in rats subjected to I/R $(n=6) .{ }^{*} p<0.05$ (significantly different from the sham group by one-way ANOVA and Tukey's post hoc test); ${ }^{* *} p<0.05$ (significantly different from the I/R group by oneway ANOVA and Tukey's post hoc test). DMSO, dimethyl sulfoxide; I/R, ischemia/ reperfusion; LDH, lactate dehydrogenase.

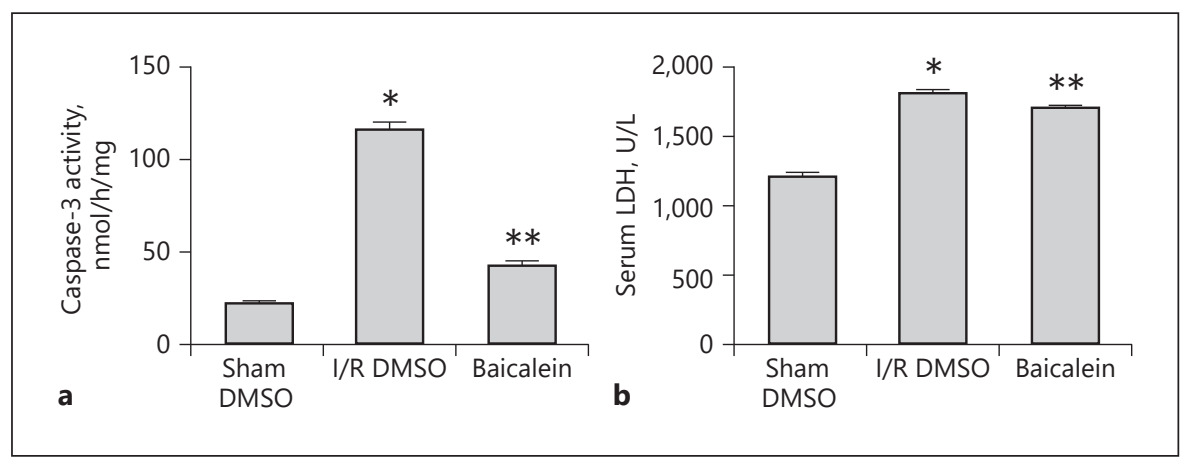

Effect of Baicalein on the TNF- $\alpha / I L-10$ Ratio. The ratio of the inflammatory marker TNF- $\alpha$ to the anti-inflammatory marker IL-10 in the livers of experimental rats was calculated. Compared to the sham group, the TNF- $\alpha / \mathrm{IL}-$ 10 ratio was significantly increased in the livers of rats in the I/R group $(p<0.05)$. Compared to the I/R group, a significant reduction in this ratio was observed in the baicalein preconditioning group $(p<0.05$; Table 2$)$.

\section{Effect of Baicalein on Oxidative Stress}

Since oxidative stress plays an important role in hepatic I/R injury and baicalein is a potent antioxidant, we examined the changes in lipid peroxidation and the level of endogenous antioxidant enzymes in the liver upon baicalein pretreatment.

Effect of Baicalein on Lipid Peroxidation. As shown in Figure 4a, in the present study, compared to the sham group, the lipid peroxidation product MDA was significantly increased in the livers of rats in the I/R group. Baicalein pre- conditioning significantly blunted the increased lipid peroxidation in the liver compared to the I/R group $(p<0.05)$.

Effect of Baicalein on Antioxidant Enzymes (MnSOD, $G P x$ ). The results in Figure $4 \mathrm{~b}$ and $\mathrm{c}$ show that the MnSOD and GPX activities were significantly reduced in the I/R group compared to the sham group. Baicalein preconditioning significantly restored the activities of endogenous antioxidant enzymes in the liver compared to the I/R group $(p<0.05)$.

\section{Effect of Baicalein on Apoptosis and Necrosis}

We next asked whether baicalein preconditioning affects apoptotic or necrotic cell death in the liver. I/R injury has been shown to increase caspase-3 activity, which in turn induces apoptotic cell death in the liver. As shown in Figure 5, caspase-3 and LDH cytolytic enzyme activities were significantly increased in the I/R group compared with the sham group. Baicalein preconditioning significantly suppressed caspase-3 activity and thus re- 
duced apoptotic cell death in the liver compared to the I/R group. Furthermore, baicalein significantly reduced LDH activity and necrotic cell death in liver tissues compared to the I/R group $(p<0.05)$.

\section{Discussion}

In this study, we found that baicalein preconditioning protects the liver against I/R injury by inhibition of ROS production, preservation of endogenous antioxidants, and suppression of inflammatory response. Moreover, our results showed that in livers subjected to $I / R$, baicalein can modulate apoptosis/necrosis and activate cell survival pathways against oxidative injury and inflammation. Those protective effects are related to increase in endogenous antioxidants, increase in anti-inflammatory cytokine, IL-10, reduction in leukocyte infiltration, and the associated increase in NF- $\kappa \mathrm{B}$ mRNA transcription and production of TNF- $\alpha$ and reduction in the TNF- $\alpha /$ IL-10 ratio.

Oxidative stress is the milestone of several liver diseases and modulates acute and chronic cell injury in the liver $[24,25]$. ROS including hydrogen peroxide, superoxide anion, and other free radicals are generated during reperfusion of ischemic liver [26]. The damage created by oxidative stress may be direct or indirect. Directly, ROS can damage hepatocytes by many mechanisms, including lipid peroxidation, DNA oxidation, and enzyme denaturation [27-29]. They also may act indirectly by acting as signaling molecules that upregulate NF- $\mathrm{BB}$ expression, activating neutrophil accumulation in the liver and releasing TNF- $\alpha$. This may lead to not only inflammation and necrosis, but also apoptosis [30]. In the present study, 30-min pretreatment with baicalein prevented loss of cell viability and reduced lipid peroxidation while preventing reduction in the activity of both MnSOD and GPx enzymes. MnSOD is considered an important antioxidant enzyme in the mitochondria which protects against oxidative stress. Previous studies showed that baicalein restored hydrogen peroxide-induced reduction in MnSOD protein expression and activity in mitochondria by activating the transcription factor NF-E2-related factor 2 (Nrf2), which is a critical regulator of $\mathrm{MnSOD}$ in mitochondria [31]. On the other hand, GPx reduces lipid hydroperoxides to their corresponding alcohols and reduces free hydrogen peroxide to water. Previous studies showed that baicalein abolished cognitive deficits in rats by increasing the activity of GPx, superoxide dismutase, and other antioxidants, enzymes, and anti-inflammatory effects [32]. Moreover, baicalein showed protection against I/R injury in cardio- myocytes via its scavenging capacity of free radicals [33]. Although we did not measure the direct free radical scavenging activity of baicalein in the present study, being a flavonoid, baicalein possess free radical scavenging and lipid peroxidase-inhibiting activity $[6,34]$. As mentioned previously, ROS during reperfusion act as signaling molecules that upregulate mRNA expression of NF- $\kappa B$. NF$\kappa \mathrm{B}$ is a transcription factor located at the cytoplasm in inactive form. During I/R, it is activated by phosphorylation and translocated to the nucleus where it induces gene expression of several genes including TNF- $\alpha$ [30]. In our study we demonstrated that following $I / R$ injury, the mRNA expression of NF- $\kappa \mathrm{B}$ is increased, which is in accordance with previous studies. We also showed that the TNF- $\alpha$ level in the liver tissues was elevated, which is a direct consequence of increased NF- $\kappa$ B. By binding with TNF- $\alpha$ type 1 receptors, TNF- $\alpha$ causes further phosphorylation and activation of NF- $\kappa \mathrm{B}$, creating a vicious cycle, and increases the extent of damage. We showed previously that during hepatic I/R injury not only the expression of TNF- $\alpha$ is increased, but also upregulation of TNF- $\alpha$ type 1 receptors takes place [35]. Activation of NF- $\kappa B$ increases the expression of ICAM-1 and other adhesion molecules that increase the recruitment of inflammatory cells to the liver following reperfusion [36]. The infiltrating cells also produces more TNF- $\alpha$ and other proinflammatory cytokines which aggravate inflammation and hepatic injury. We showed in our study that there was an increase in inflammatory cell infiltration following I/R that confirmed the previous reports. Although TNF- $\alpha$ plays an important role in $\mathrm{I} / \mathrm{R}$ injury in the liver, we showed in our previous study that it is not the only player in mediating hepatic damage and that neutralizing TNF- $\alpha$ by infliximab, a monoclonal antibody, partially protects against the deleterious effects of I/R [35].

IL-10 is an anti-inflammatory cytokine. It produces its anti-inflammatory effects by binding to IL-10 receptors, which induces STAT3 signaling with subsequent increases in cell survival [37]. Previous studies showed that IL-10 suppresses NF- $\kappa \mathrm{B}$ signaling and promotes hepatic cell survival $[38,39]$. Reduction in anti-inflammatory cytokines such as IL-10 seems to play a crucial role in I/Rinduced hepatic damage. We demonstrated in the present study that $\mathrm{I} / \mathrm{R}$ was associated with a reduction in the level of IL-10 in hepatic tissues. A recent study showed that exogenous administration of IL-10 increased the survival of ob/ob animals at $24 \mathrm{~h}$ after I/R and significantly decreased serum ALT levels [40]. In our study it seems that the downregulation of NF- $\kappa \mathrm{B}$ by baicalein was mainly mediated via the elevation of IL-10 level. This may be re- 
sponsible for the reduction in TNF- $\alpha$ level and neutrophil infiltration observed in baicalein-treated rats. I/ $\mathrm{R}$ also increases the TNF- $\alpha / \mathrm{IL}-10$ ratio, indicating an imbalance between inflammatory and anti-inflammatory cytokines favoring hepatic inflammation and cell damage. Baicalein preconditioning reverses these deleterious changes and decreased the TNF- $\alpha /$ IL-10 ratio. A recent study showed that baicalein pretreatment protects mice against I/ $\mathrm{R}$ injury by inhibition of the NF- $\kappa B$ pathway, which confirmed our present findings [16]. Previous studies did not investigate the effect of baicalein, anti-inflammatory cytokines, or oxidant signaling in I/R models. In our study we demonstrated that baicalein preconditioning using a $300 \mathrm{mg} / \mathrm{kg}$ dose not only suppressed the hepatic inflammation following $\mathrm{I} / \mathrm{R}$, but also alleviated oxidative stress, restored endogenous antioxidants, and increased the anti-inflammatory cytokine IL-10.

Apoptosis and necrosis are central mechanisms of cell death in liver IR injury that directly indicate cell death conditions. This study analyzed apoptotic cells in ischemic liver $1 \mathrm{~h}$ after reperfusion. Histopathological examination of liver tissues showed hepatocyte degeneration and pyknosis following I/R. Then, the caspase-3 activity of liver tissue was also examined $1 \mathrm{~h}$ after reperfusion, which also reflected the condition of apoptotic cells in ischemic liver. In line with the histopathological examination, I/R significantly increased caspase- 3 activity after reperfusion. We also showed that the cytolytic enzyme activity LDH was increased following reperfusion, indicating cytolysis. Baicalein preconditioning inhibits caspase- 3 as well as LDH activity and reduces pyknosis and hepatocyte degeneration. The antiapoptotic effect of baicalein may be attributed to its antioxidant and anti-inflammatory effects.

Taken together, the present study provides evidence that baicalein preconditioning exerts hepatoprotective effects through inhibiting inflammatory and oxidant signal- ing and promoting IL-10 in a rat model of hepatic I/R injury. Baicalein could be a promising drug for patient having undergone liver transplantation in clinical settings.

\section{Acknowledgments}

The authors would like to thank Prof. L. Rashed (Biochemistry Department, Faculty of Medicine, Cairo University, Egypt) for assistance in performing the PCR technique and Dr. Dr. A.A. AbuelAtta, Faculty of Veterinary Medicine, Zagazig University for histopathological examination of livers.

\section{Statement of Ethics}

The protocol was approved by the institutional animal care and use committee and the local experimental ethics committee. The authors have no ethical conflicts to disclose.

\section{Disclosure Statement}

The authors have no conflicts of interest to declare.

\section{Funding Sources}

This work did not receive any specific grant from funding agencies in the public, commercial, or not-for-profit sectors.

\section{Author Contributions}

S. Gamal contributed to the experimental design, performed the experiments, conceived the study, oversaw all data collection and analysis, and drafted the manuscript. H.M. El-Fayoumi contributed to the experimental design and manuscript preparation. M.F. Mahmoud contributed to the experimental design, data analysis and interpretation, and manuscript preparation. All authors read and approved the final manuscript.

\section{References}

1 Lentsch AB, Kato A, Yoshidome H, McMasters KM, Edwards MJ. Inflammatory mechanisms and therapeutic strategies for warm hepatic ischemia/reperfusion injury. Hepatology. 2000 Aug;32(2):169-73.

2 Jaeschke H. Molecular mechanisms of hepatic ischemia-reperfusion injury and preconditioning. Am J Physiol Gastrointest Liver Physiol. 2003 Jan;284(1):G15-26.

3 Zapolska-Downar D, Naruszewicz M. Propionate reduces the cytokine-induced VCAM-1 and ICAM-1 expression by inhibiting nuclear factor-kappa B (NF-kappaB) activation. J Physiol Pharmacol. 2009 Jun;60(2):123-31.
4 Okaya T, Lentsch AB. Cytokine cascades and the hepatic inflammatory response to ischemia and reperfusion. J Invest Surg. 2003 May-Jun;16(3):141-7.

5 van Leyen K, Kim HY, Lee SR, Jin G, Arai K, Lo EH. Baicalein and 12/15-lipoxygenase in the ischemic brain. Stroke. 2006 Dec;37(12): 3014-8.

6 Shieh DE, Liu LT, Lin CC. Antioxidant and free radical scavenging effects of baicalein, baicalin and wogonin. Anticancer Res. 2000 Sep-Oct;20(5A):2861-5.
7 Gao Z, Huang K, Yang X, Xu H. Free radical scavenging and antioxidant activities of flavonoids extracted from the radix of Scutellaria baicalensis Georgi. Biochim Biophys Acta. 1999 Nov;1472(3):643-50.

8 Chang WS, Lee YJ, Lu FJ, Chiang HC. Inhibitory effects of flavonoids on xanthine oxidase. Anticancer Res. 1993 Nov-Dec;13(6A):216570.

9 Chen ZY, Su YL, Bi YR, Tsang SK, Huang Y. Effect of baicalein and acetone extract of Scutellaria baicalensis on canola oil oxidation. J Am Oil Chem Soc. 2000;77(1):73-8. 
10 Hsieh CJ, Hall K, Ha T, Li C, Krishnaswamy G, Chi DS. Baicalein inhibits IL-1beta- and TNF-alpha-induced inflammatory cytokine production from human mast cells via regulation of the NF-kappaB pathway. Clin Mol Allergy. 2007 Nov;5(1):5.

11 Hwang YS, Shin CY, Huh Y, Ryu JH. Hwangryun-Hae-Dok-tang (Huanglian-JieDu-Tang) extract and its constituents reduce ischemia-reperfusion brain injury and neutrophil infiltration in rats. Life Sci. 2002 Sep; 71(18):2105-17.

12 Lee HH, Yang LL, Wang CC, Hu SY, Chang SF, Lee YH. Differential effects of natural polyphenols on neuronal survival in primary cultured central neurons against glutamateand glucose deprivation-induced neuronal death. Brain Res. 2003 Oct;986(1-2):103-13.

13 Sharma V, Mishra M, Ghosh S, Tewari R, Basu A, Seth P, et al. Modulation of interleukin-1beta mediated inflammatory response in human astrocytes by flavonoids: implications in neuroprotection. Brain Res Bull. 2007 Jun; 73(1-3):55-63.

$14 \mathrm{Wu} \mathrm{K}, \mathrm{Li} \mathrm{H}$, Tian J, Lei W. Protective effect of baicalein on renal ischemia/reperfusion injury in the rat. Ren Fail. 2015 Mar;37(2):285-91.

$15 \mathrm{Wu}$ YL, Lian LH, Wan Y, Nan JX. Baicalein inhibits nuclear factor- $\kappa \mathrm{B}$ and apoptosis via c-FLIP and MAPK in D-GalN/LPS induced acute liver failure in murine models. Chem Biol Interact. 2010 Dec;188(3):526-34.

16 Liu A, Huang L, Fan H, Fang H, Yang Y, Liu S, et al. Baicalein pretreatment protects against liver ischemia/reperfusion injury via inhibition of NF- $\mathrm{KB}$ pathway in mice. Int Immunopharmacol. 2015 Jan;24(1):72-9.

17 Aggarwal BB, Vilček J, editors. Tumor necrosis factor: structure, function and mechanism of action. New York: Marcel Dekker; 1992.

18 Moore KW, Vieira P, Fiorentino DF, Trounstine ML, Khan TA, Mosmann TR. Homology of cytokine synthesis inhibitory factor (IL$10)$ to the Epstein-Barr virus gene BCRFI. Science. 1990 Jun;248(4960):1230-4

19 Cho S, Choi Y, Park S, Park T. Carvacrol prevents diet-induced obesity by modulating gene expressions involved in adipogenesis and inflammation in mice fed with high-fat diet. J Nutr Biochem. 2012 Feb;23(2):192201.
20 Nishikimi M, Appaji N, Yagi K. The occurrence of superoxide anion in the reaction of reduced phenazine methosulfate and molecular oxygen. Biochem Biophys Res Commun. 1972 Jan;46(2):849-54.

21 Ohkawa H, Ohishi N, Yagi K. Assay for lipid peroxides in animal tissues by thiobarbituric acid reaction. Anal Biochem. 1979 Jun;95(2): 351-8.

22 Hayami S, Ikeda K, Sun F, Tanaka K, Kojo S. Increase of caspase- 3 activity in rat liver and plasma by thioacetamide. Biochem Pharmacol. 1999 Dec;58(12):1941-3.

23 Deng J, Feng J, Liu T, Lu X, Wang W, Liu N, et al. Beraprost sodium preconditioning prevents inflammation, apoptosis, and autophagy during hepatic ischemia-reperfusion injury in mice via the P38 and JNK pathways. Drug Des Devel Ther. 2018 Nov;12:4067-82.

24 Li M, Wang S, Li X, Kou R, Wang Q, Wang $\mathrm{X}$, et al. Diallyl sulfide treatment protects against acetaminophen-/carbon tetrachloride-induced acute liver injury by inhibiting oxidative stress, inflammation and apoptosis in mice. Toxicol Res (Camb). 2018 Oct;8(1): 67-76.

25 Lemasters JJ, Nieminen AL, Qian T, Trost LC Elmore SP, Nishimura Y, et al. The mitochondrial permeability transition in cell death: a common mechanism in necrosis, apoptosis and autophagy. Biochim Biophys Acta. 1998 Aug;1366(1-2):177-96.

26 Urakami H, Abe Y, Grisham MB. Role of reactive metabolites of oxygen and nitrogen in partial liver transplantation: lessons learned from reduced-size liver ischaemia and reperfusion injury. Clin Exp Pharmacol Physiol. 2007 Sep;34(9):912-9.

27 Jaeschke H. Role of reactive oxygen species in hepatic ischemia-reperfusion injury and preconditioning. J Invest Surg. 2003 May-Jun; 16(3):127-40

28 Madesh M, Hajnóczky G. VDAC-dependent permeabilization of the outer mitochondrial membrane by superoxide induces rapid and massive cytochrome c release. J Cell Biol. 2001 Dec;155(6):1003-15.

29 Zhao K, Zhao GM, Wu D, Soong Y, Birk AV, Schiller PW, et al. Cell-permeable peptide antioxidants targeted to inner mitochondrial membrane inhibit mitochondrial swelling, oxidative cell death, and reperfusion injury. J Biol Chem. 2004 Aug:279(33):34682-90.
30 Llacuna L, Bárcena C, Bellido-Martín L, Fernández L, Stefanovic M, Marí M, et al. Growth arrest-specific protein 6 is hepatoprotective against murine ischemia/reperfusion injury. Hepatology. 2010 Oct;52(4):1371-9.

31 D'amico R, Fusco R, Gugliandolo E, Cordaro M, Siracusa R, Impellizzeri D, et al. Effects of a new compound containing palmitoylethanolamide and baicalein in myocardial ischaemia/reperfusion injury in vivo. Phytomedicine. 2019 Feb;54:27-42.

32 Mao X, Cao Y, Li X, Yin J, Wang Z, Zhang Y, et al. Baicalein ameliorates cognitive deficits in epilepsy-like tremor rat. Neurol Sci. 2014 Aug;35(8):1261-8.

33 Chang WT, Shao ZH, Yin JJ, Mehendale S, Wang CZ, Qin Y, et al. Comparative effects of flavonoids on oxidant scavenging and ischemia-reperfusion injury in cardiomyocytes. Eur J Pharmacol. 2007 Jul;566(1-3):58-66.

34 Hara H, Sukamoto T, Ohtaka H, Abe K, Tatumi Y, Saito Y, et al. Effects of baicalein and alpha-tocopherol on lipid peroxidation, free radical scavenging activity and 12 -O-tetradecanoylphorbol acetate-induced ear edema. Eur J Pharmacol. 1992 Oct;221(2-3):193-8.

35 Mahmoud MF, El Shazly SM, Barakat W. Inhibition of TNF- $\alpha$ protects against hepatic ischemia-reperfusion injury in rats via NF- $\kappa \mathrm{B}$ dependent pathway. Naunyn Schmiedebergs Arch Pharmacol. 2012 May;385(5):465-71.

36 Jaeschke H, Smith CW. Cell adhesion and migration. III. Leukocyte adhesion and transmigration in the liver vasculature. Am J Physiol. 1997 Dec;273(6):G1169-73.

37 Mosser DM, Zhang X. Interleukin-10: new perspectives on an old cytokine. Immunol Rev. 2008 Dec;226(1):205-18.

38 Schottelius AJ, Mayo MW, Sartor RB, Baldwin AS Jr. Interleukin-10 signaling blocks inhibitor of kappaB kinase activity and nuclear factor kappaB DNA binding. J Biol Chem. 1999 Nov;274(45):31868-74.

39 Osborn O, Olefsky JM. The cellular and signaling networks linking the immune system and metabolism in disease. Nat Med. 2012 Mar;18(3):363-74.

40 Sutter AG, Palanisamy AP, Ellet JD, Schmidt MG, Schnellmann RG, Chavin KD. Interleukin-10 and Kupffer cells protect steatotic mice livers from ischemia-reperfusion injury. Eur Cytokine Netw. 2014 Oct-Dec;25(4):69-76. 